Teknokultura. Revista de Cultura Digital y Movimientos Sociales

ISSNe: $1549-2230$

http://dx.doi.org/10.5209/TEKN.71514

\title{
Blockchain para la transparencia, gestión pública y colaboración ${ }^{1}$
}

\author{
Lucía Benítez-Eyzaguirre²
}

Recibido: 14 de septiembre de 2020 / Aceptado: 1 de diciembre de 2020 Open peer reviews

Resumen. Se describen aquí las aplicaciones innovadoras de blockchain para la gobernanza y otros modelos económicos, de cara al emprendimiento social en la resolución de problemas de ámbito local, dentro de las iniciativas de la colaboración público-privada propias del Gobierno Abierto, así como los riesgos identificados y los efectos no contemplados del modelo propuesto.

Palabras clave: emprendimiento social; innovación social; tecnología de la comunicación.

\section{[en] The role of blockchain in transparency, public management and collaboration}

Abstract. This paper describes innovative applications of blockchain technologies for governance and other economic models. The paper focuses on the application of this technology in the context of social entrepreneurship and as a solution to local issues in public-private initiatives that are a characteristic of Open Government. The article also addresses unidentified risks and unnoticed effects of blockchain applications in these domains.

Keywords: communication technologies; social entrepreneurship; social innovation.

Sumario. 1. Introducción. 2. Tecnologías blockchain. 3. Blockchain y software libre. 4. Gobernanza, coordinación y acuerdos institucionales sobre participación, voto y gestión. 5. Modelo económico. 6. Ciudadanía, servicios ciudadanos y Tercer sector. 7. Riesgos y efectos no contemplados. 8. Referencias.

Cómo citar: Benítez-Eyzaguirre, L. (2021). Blockchain para la transparencia, gestión pública y colaboración. Teknokultura. Revista de Cultura Digital y Movimientos Sociales, 18(1), 23-32.

\section{Introducción}

En estas páginas se desarrolla un modelo teórico para la aplicación de blockchain en una iniciativa integral de innovación social, a partir de un modelo de gobernanza inclusiva para la coproducción de servicios orientados a la creación de empleo y la promoción del emprendimiento para la puesta en marcha de iniciativas empresariales. Mientras en instituciones tradicionales, la gobernanza o conjunto de reglas y normas, así como las acciones, se determinan a través de un agente principal -que podría actuar en su propio beneficio- que toma decisiones que afectan al resto de los miembros, en blockchain las tareas de supervisión se pueden delegar en redes descentralizadas inmutables, muy confiables y seguras, que contribuyen a crear confianza en el sistema.

El proyecto pretende dar respuesta a retos clave para la dinamización económica, y para combatir pro- blemáticas sociales como las que se concretan a continuación:

1. Reducir el paro estructural y aflorar empleo no declarado e integrar a colectivos socialmente vulnerables para abordar las oportunidades de integración sociolaboral que ofrece el emprendimiento social.

2. Fomentar la innovación en la red productiva existente, consolidando el tejido empresarial ante nuevas actividades generadoras de empleo.

3. Diseñar un marco de coordinación público-privado desde el enfoque de la gobernanza de las políticas de empleo, la economía social y la innovación.

4. Rentabilizar los recursos naturales, los culturales y las oportunidades que ofrecen para la economía y el emprendimiento social (economía verde y azul, comercio, industrias creativas y culturales).

\footnotetext{
Este artículo es producto del proyecto de Investigación titulado "Ciberactivismo, Ciudadanía Digital y Nuevos Movimientos Urbanos" (CiberMov), referencia CSO2016-78386-P, financiado por el Programa Estatal de Fomento de la Investigación Científica y Técnica de Excelencia, Subprograma Estatal de Generación de Conocimiento del Ministerio de Economía, Industria y Competitividad y coordinado por el Grupo Interdisciplinario de Estudios en Comunicación, Política y Cambio Social (COMPOLÍTICAS) de la Universidad de Sevilla. Asimismo, el proyecto está cofinanciado con fondos FEDER. http://www.idi.mineco.gob.es/stfls/eSede/Ficheros/2016/Anexo_I_Ayudas_Concedidas_Proyectos_Excelencia_2016.pdf

Universidad de Cádiz (España)

E-mail: Lucia.benitez@uca.es
} 


\section{Tecnologías blockchain}

Blockchain como tecnología informática permite la transmisión, almacenamiento y confirmación de datos o información en una organización autónoma a partir del consenso entre los participantes, sobre la base constituida por dos grupos de tecnologías; por un lado, las infraestructuras de clave pública o Public Key Infrastructure (PKI) para el cifrado asimétrico y, por otro, los sistemas y algoritmos distribuidos para la toma de decisiones por consenso entre un grupo de agentes descentralizados (Preukschat, 2017).

La base de datos sobre la que opera un blockchain es descentralizada, ya que se distribuye entre todos los participantes de una red entre iguales o peer-to-peer ( $\mathrm{P} 2 \mathrm{P})$. La distribución de la información se hace a partir del consenso entre los actores y la red se estructura como una organización autónoma democrática, con aplicaciones posibles en muy diversos campos. Su aplicación a la participación ciudadana se ha concretado en el sistema de voto para la producción del consenso que describe Arteaga (2018, p. 153-156) como un sistema participativo y seguro.

Su configuración y funcionamiento pueden ser transparentes, ya que la red muestra los intercambios y transacciones de diferentes tipos de forma abierta y segura, sin precisar de intermediarios que validan las transferencias. De Filippi (2017) habla de transparencia con seudonimidad para las transacciones en un blockchain, dado que cada usuario y cada nodo quedan identificados por una dirección numérica única, seudónima de una identidad que, debido a las características técnicas de la PKI, no se puede conocer si el usuario así lo decide. Todas las transacciones quedan registradas de forma inmutable en la base de datos distribuida, es decir, un conjunto significativo de los nodos participantes guarda copias cifradas de cada transacción antes consensuada, de forma que es técnicamente imposible eliminar o falsear el registro histórico de las transacciones ejecutadas (Brandom, 2019). Esta característica de la propia naturaleza de la tecnología blockchain puede verse como una ventaja (dado que es técnicamente imposible falsear o eliminar un registro) o como una desventaja (pues también es técnicamente imposible, por ejemplo, implementar el llamado "derecho al olvido").

Blockchain puede revolucionar muchas de las tareas al prescindir de intermediarios y evitar el control individual centralizado del sistema o que el poder de la autenticidad y el valor de las transacciones (incluidas las económicas) estén en unas pocas manos. Dichas tareas pueden ser sustituidas por algoritmos automatizados o smart contracts que garanticen el valor o los efectos de una determinada transacción, según las normas y acciones programadas en dicho contrato. Los contratos inteligentes y las aplicaciones distribuidas que funcionan sobre un blockchain pueden ser la base de organizaciones descentralizadas autónomas o DAO (Decentralized Autonomous Organization). Una DAO debe tener una constitución que determine su gobernanza públicamente en el blockchain, así como un mecanismo para financiar sus operaciones (por ejemplo, a través de crowdfunding).
Un blockchain consta de:

1. Un ledger o libro de registro que permanece de forma inmutable con constancia pública -en su caso- de los cambios y transacciones que se han ido produciendo en la red. Existen también blockchain privados, mixtos y de consorcio, pero dado el ámbito de aplicación que se muestra aquí, sólo se contempla el desarrollo público del consenso. Normalmente estamos acostumbrados a que los libros de registro, independientemente de si son de acceso público o no, sean propiedad o estén bajo control de alguien, de un solo agente o institución, pero no de aquellos que lo utilizan para publicar algo en él. En el caso de blockchain, el ledger no es de nadie, sino que es de todos a la vez, de todos los que quieren publicar algo en el registro.

2. Unos tokens digitales que permite el acceso y el seguimiento de las actividades realizadas en el blockchain. Los mensajes que se intercambian representan transacciones sobre activos, bienes o servicios, compras, alquileres, contribuciones sociales, ventas, y una amplia gama de operaciones que puedan imaginarse. Los tokens se transmiten de un agente a otro en mensajes encriptados con un sistema de clave pública o asimétrica, que hace que solo el destinatario de dicho mensaje pueda descifrarlo. Por tanto, no se revela su contenido a terceros, a pesar de tener cualquiera acceso a los mensajes distribuidos por todos los nodos del blockchain. La implementación de tokens que ha tenido mayor éxito son las criptomonedas digitales como el bitcoin, pero no queda limitada a dicho uso. La ONU utiliza un sistema de identidad en los campamentos de refugiados sirios Azraq y Zaatari para ofrecer documentación a las personas que la perdieron en su huida, pero también para bridar ayuda humanitaria y control los efectos de la Covid, que se apoya en la tecnología de IrisGuard (2020). En este caso, un token puede ser la identidad de estas personas.

3. Bloques que agrupan las transferencias de tokens y que se generan periódicamente y de forma sucesiva cada vez que se completa una transacción. La transacción siguiente a una dada quedaría enlazada a la anterior, por lo que el conjunto de todas las transferencias queda estructurada en una cadena global de bloques (el blockchain). El cifrado de cada eslabón de esa cadena de bloques de transacciones, teniendo en cuenta el histórico de transacciones anteriores en lugar de cifrar cada bloque individualmente, hace materialmente imposible falsear una transacción realizada en un momento que refleja el registro histórico de todas las transacciones realizadas. Es decir, para poder suprimir o falsear una transacción, habría que hacerlo sobre todo el histórico de transacciones que sucedieran a dicha transacción, que resulta inviable en cadenas de blockchain de gran tamaño. 
Al introducir nuevos bloques, blockchain propone un reto matemático a cada uno de los agentes o nodos o proof of work, para el que debe estimarse una recompensa por el trabajo de minar datos como un estímulo a la participación. La primera de las soluciones suministrada por un nodo se acepta por todos, al comprobar que es correcta, y se integra en la cadena de bloques, junto a la información que ha llevado a esa solución, con el certificado imborrable y no manipulable del momento en que se realizó la operación y ésta quedó validada por consenso. La formulación matemática, la transparencia, la seguridad y la auditoría permanente de las partes se mantienen junto con la garantía de la encriptación de la información.

Por una parte, los algoritmos que ejecutan las transacciones en un blockchain tienen un coste computacional enormemente elevado y suponen un consumo energético gigantesco, por lo que aún no se prestan bien para aplicaciones que requieran el tiempo de respuesta de un sistema interactivo (de menos de unos pocos segundos) en la espera a finalizar una transacción. Por ejemplo, la velocidad del blockchain de bitcoin es de una transacción por segundo (tps), pudiendo alcanzar como máximo picos de 7 tps. Comparado con las transacciones de tarjetas de crédito VISA (de 2.000 a 10.000 tps) y twitter (de 5.000 a 15.000 tps), la velocidad de las aplicaciones de un blockchain es ínfima, lo que dificulta muchas de las utilidades transaccionales previstas. Este aspecto afecta a la escalabilidad de las transacciones ya que puede producir esperas y retrasos y minar su adopción.

Por otra parte, los algoritmos de cifrado y las infraestructuras de clave pública garantizan automáticamente la veracidad de cualquier transacción, sin necesidad de ningún agente que la valide. Cualquier participante en el blockchain puede averiguar la validez de cualquier transacción en cualquier momento a lo largo de toda su historia. Para que blockchain consolide su capacidad disruptiva, la regulación del sistema será un elemento clave y se basa en la gobernabilidad que debe ser adoptada por los miembros en un proceso democrático y abierto como el que se ha experimentado en Alcobendas (Arteaga, 2018).

\section{Blockchain y software libre}

El sistema de transacción de blockchain utiliza una Interfaz de Desarrollo de Aplicaciones o Application Programming Interface (API) abierta para tener acceso al conjunto de funciones que presta. Las API permiten la comunicación entre los diferentes componentes del software y se utilizan como parte de las bibliotecas de programación de forma que los programadores y desarrolladores simplifican su trabajo empleando sus desarrollos y cumpliendo con el principio de la actitud hacker, propuesta por Raymond (2001), de que "ningún problema tendría que resolverse dos veces" sino que ese tiempo se debe emplear en la mejora de tus proyectos o en otros que atiendan necesidades humanas o sociales más relevantes.
Las API son imprescindibles para el desarrollo de blockchain (Preukschat, 2017, p. 35), de la misma forma que la mayoría de blockchain se ubica dentro del software libre, entendiendo como tal aquel que garantiza las cuatro libertades básicas (libertad de uso, libertad de lectura y estudio del código, la libertad para su modificación y la libertad de distribución) (Stallman, 2004). Esto supone la apertura del código y el derecho de acceso al mismo, o sea, un gran nivel de transparencia y mayor solidez y calidad, pero también facilita la interoperabilidad (la capacidad del sistema para intercambiar y usar información con objetivos comunes y bajo consenso con la finalidad de obtener beneficios mutuos). El software libre es viable y más sostenible en valores económicos y sociales, incluso a veces de mayor calidad que el privativo, ya que la participación de un colectivo en su desarrollo lo convierte en más sólido, más innovador y versátil, gracias al recurso continuo al conocimiento público (Stallman, 2004).

Cuando blockchain se desarrolla como pública, los usuarios y participantes reciben el conjunto de libertades sobre el que se sustenta el software libre pero las garantías y el respeto a la libertad no facilitan necesariamente el logro de una solución viable ni de ventaja económica, así como tampoco la adopción de la tecnología que es a fin de cuentas una cuestión cultural. Por ello, cuando blockchain busca la ventaja competitiva y/o la monetización su desarrollo puede ser privado e incluir límites en el acceso al código fuente o restricciones de uso en determinados supuestos, por lo que no pueden considerarse de código libre.

En concreto, en sus inicios, la licencia de la mayor parte del software que sirve para implementar blockchain es la del Massachusetts Institute of Technology (MIT), muy abierta, ya que no sólo cumple los requisitos del software libre, sino que se adapta a otras posibilidades, porque no tiene copyright, y a su modificación. Permite la redistribución del software con fines comerciales, las sublicencias o la mezcla con otros códigos fuente de diferentes licencias. La garantía del open source ha aportado transparencia para la credibilidad en el sistema, pero a la vez su carácter abierto ha posibilitado el desarrollo de versiones privadas y semipúblicas de blockchain, y todo un repertorio de licencias sobre las diferentes soluciones que aporta esta tecnología. Por otra parte, la combinación de blockchain con el sistema de API se ha transformado en el respaldo de muchos negocios, especialmente del sistema bancario y financiero, pero también mejora los desarrollos para la gestión pública.

\section{Gobernanza, coordinación y acuerdos institucionales sobre participación, voto y gestión}

El concepto de res publica, en su sentido original de la expresión latina, no sólo hace referencia a la cosa pública sino que también abarca a las regulaciones y resoluciones del pueblo, como un asunto de interés compartido y público (Garcés, 2011). Así, en su dimensión digital, se entendería como el debate públi- 
co y los derechos de participación en función de las características de la configuración política del espacio virtual donde se desarrollan, un aspecto para el que la tecnología blockchain presenta un enorme potencial innovador. Si entendemos blockchain como el espacio público digital para el ejercicio de la gobernanza, su institucionalización y su responsabilidad formaría parte del procomún: "la necesidad de entender la gobernanza electrónica como parte integrante de la esfera de lo político en la actualidad es lo que ha llevado a definir una ampliación de los derechos humanos en los digitales" (Alonso y Lafuente, 2011).

Los principios de una gobernanza de calidad, según aprobó en Consejo la Confederación Española de Organizaciones Empresariales (CEOE) en 2014, están relacionados con la coordinación entre las diferentes entidades, públicas y privadas, los niveles de gobierno, los distintos sectores y la coordinación horizontal de carácter territorial. También se valora el fortalecimiento de las capacidades y habilidades de cada uno de los niveles de gobierno, así como las condiciones para que las inversiones sean efectivas en materia de transparencia, regulación y eficacia de la compra pública. Todas estas características forman parte de los principios del gobierno abierto, que surge de la adopción de la filosofía del software libre a los principios de la democracia, en el que la ciudadanía y los agentes contribuyen a la mejora de los servicios públicos. Sus pilares son precisamente la transparencia, la participación y la colaboración. De forma más concreta, el sistema democrático de un gobierno abierto supone el acceso libre y gratuito a datos abiertos, sistemas para implementar la transparencia, participación y colaboración en los procesos, así como un soporte tecnológico que garantice el acceso a la información. El gobierno abierto es "aquel que entabla una constante conversación con los ciudadanos con el fin de escuchar lo que ellos dicen y solicitan, que toma decisiones basadas en sus necesidades y teniendo en cuenta sus preferencias" (Calderón y Lorenzo, 2010), por ello facilita la colaboración entre todos los sectores y fomenta la comunicación.

La orientación hacia esos principios se puede realizar mediante el uso de blockchain en la organización y gestión de la iniciativa, ya que en sí misma esta tecnología facilita el logro de los objetivos. Jun (2018), que ha localizado más de 100 proyectos de blockchain que se han desarrollado en unos 30 países, considera esta tecnología propia del consenso y el consenso forma parte de la humanidad. Jun (2018) sistematiza los cinco principios de respeto cuando se sustituye la burocracia por la cadena de bloques: la legislación sobre el estatuto de blockchain, la divulgación con transparencia del código fuente y los datos, la incorporación de la administración autónoma, la creación de un sistema de gobernanza de democracia directa y la elaboración de un Sistema de Gobierno (DAG).

La gobernanza en un blockchain comienza en la negociación democrática y horizontal sobre el contrato de fundación que blinda el modelo innovador, descentralizado, distribuido y sin intermediarios. Permite la creación de un sistema de votación transparente y de- mocrático no sólo para las decisiones constituyentes de la organización, sino también para los procesos que la requirieran. Un modelo experimental de desarrollo en el municipio madrileño de Alcobendas ha ensayado las bases comunicativas y técnicas, así como los requisitos (Arteaga, 2018).

Como modelo político y de gobernanza resulta disruptivo, porque su sistema es transparente y fomenta la creación de la confianza social, elimina la corrupción y prescinde de intermediarios - por tanto, también de costes- en un prototipo que es potencialmente replicable. El intercambio de datos en que la transferencia de valor se realiza a través de contratos inteligentes, de forma horizontal entre los participantes. Todo ello va acompañado del ahorro, la inmediatez, el aumento de la seguridad, de la flexibilidad y optimización en los procesos. En paralelo, se logra la integración de las administraciones mientras se respeta el cumplimiento de la ley y las normas en los intercambios financieros y de activos, la gestión de los contratos e identidades, los registros de propiedad y los derechos de autor, la participación ciudadana, entre otros. La Unión Europea viene analizando la aplicación de blockchain a través de un observatorio el European Union Blockchain Observatory \& Forum (EUBOF) y una serie de informes sobre características y garantías (EUBOF, 2018).

Como decíamos antes, los smart contracts o contratos inteligentes se autoejecutan en cuanto se cumplen las condiciones que proponen las partes en el acuerdo institucional. Esta garantía no sólo elimina trámites burocráticos, sino que evita el fraude y reduce la intervención de evaluadores. Todo ello repercute en beneficios para administraciones, empresas y ciudadanía.

A fin de cuentas, la gestión burocrática se resume en la constancia en un ledger en el que se puede confirmar de manera directa todos los registros y hechos inalterables. Su valor como garantía pública puede considerarse superior debido al cifrado criptográfico asimétrico que combina una clave privada y una pública. Una clave pública no permite conseguir ninguna información sobre la clave privada ni sobre el contenido protegido. La clave privada es la única que permite: (a) acceder a una cierta información cifrada y destinada al propietario de dicha clave; y (b) certificar una cierta información de modo que queda garantizado que solo el propietario de dicha clave ha podido hacerlo. Mientras, la clave pública permite a un tercero: (c) enviar con seguridad una información cifrada con la garantía de que sólo el destinatario la podrá descifrar; y (d) comprobar con transparencia que la información protegida proviene realmente de quien dice ser.

Para atender desde las instituciones públicas estos cambios hay que prestar atención a la selección de un caso real adecuado por su impacto en el sector público y en la industria ya que blockchain genera valor a través de las redes con facilidad para su transferencia y unos costos muy reducidos, gracias a que es un sistema de confianza. Su aplicación práctica y el logro de una solución comienza estableciendo el consenso de la comunidad, la estructura y gobernanza de los smart contracts, 
contando para ello con un diseño para las interacciones entre actores, así como del modelo de confianza.

Los requisitos técnicos de la gestión de la administración pública deben corresponderse con el diseño tecnológico, su arquitectura técnica, la integración entre elementos externos e internos, los requisitos de normas y su cumplimiento, y las transacciones por segundo. De esta forma se busca que el blockchain sea sólido gracias a la concreción de los sistemas de transacción, de confianza y de propiedad para evitar que el sistema elimine procesos repetitivos o duplicados, para que genere confianza como una cualidad más añadida al consenso, la determinación de la procedencia de las interacciones y la inmutabilidad del sistema.

\subsection{Desarrollo de normas}

El desarrollo legislativo a través de la colaboración es una de las vías de la participación ciudadana en el ejercicio del gobierno abierto. Por esta vía se concretan los planes de acción tanto a la hora de priorizar medidas como de comentar o debatir sobre determinados repositorios legales. Para ello, se viene utilizando GitHub como herramienta abierta y muy experimentada en el mundo del software libre, porque es versátil: certifica un control de cambios capaz de realizar el seguimiento de la edición de una legislación. GitHub, como sitio de hosting de código colaborativo, cuenta con una interfaz gráfica y de escritorio -también para móvil- con un diseño muy social que facilita el seguimiento tanto de los repositorios como de los usuarios, recibir actualizaciones de los últimos cambios y descubrir nuevos proyectos. El sistema de control de los cambios se realiza con un Version Control System (VCS) y con la división del texto en diferentes fragmentos (a nivel de caracteres, líneas, párrafos, etc.) No se generan diferentes versiones del texto, sino que se guardan instantáneas (snapshots) de toda la legislación en cada momento. Además, si se cuenta con un repositorio común, facilita la colaboración y permite el acceso independiente, ya que cada usuario puede decidir dónde contribuir.

Las características de GitHub como sistema open source permiten su uso sin restricciones, así como las modificaciones para aplicaciones concretas como la legislación (Wright y De Filippi, 2015). Se puede migrar a cualquier otro sistema con independencia de su desarrollo -y así contar con la posibilidad de reutilizar toda la información a través de un clonado-, $\mathrm{y}$ permite acceder a la legislación vigente y a la derogada.

Respecto a GitHub o GitLaw (Voelker, 2012), blockchain supone ventajas adicionales porque los registros son inalterables, el control es colectivo y no es de un solo usuario, elimina la duplicidad de versiones; todos pueden añadir, modificar y quitar contenidos sin necesidad de autorización alguna ya que, si la transacción es válida y deja al blockchain en un estado consistente, ésta queda reflejada. Para la realización práctica de estas ini- ciativas existe la aplicación CrowdLaw (The GovLab, 2017).

\subsection{Contratación, gestión de presupuestos y de financiación}

El ámbito de la contratación, gestión de presupuestos y la financiación llevan sistemas complejos de control y cumplimiento normativo que resultan costosos y vulnerables, dado el nivel de fraude y corrupción que alcanza a las instituciones públicas. La ventaja del sistema se encuentra también en que la fortaleza de la información obtenida a través de blockchain permite que las decisiones se tomen de forma consensuada, con la participación activa de cada agente, sin intermediarios, con transparencia y rendición de cuentas, todo ello facilita la labor de las administraciones. Sin ser probablemente la mejor de las opciones actuales, blockchain aporta una gran transparencia al sistema, como se ha dicho.

\subsection{Auditoría económica}

La transparencia de blockchain puede evitar la realización de auditorías ya que facilita la comprobación de los datos incluso cuando la información se actualiza o en el mismo momento en que se comparte, una transparencia que podría alertar sobre irregularidades. Sin embargo, la aplicación práctica de auditorías, tal y como las conocemos, se encuentra con los frenos de la seguridad y complejidad del sistema. Su ventaja es que blockchain protege de riesgos derivados de la aplicación de normativas, así como asegura el tiempo y reduce los costes de estas gestiones.

\section{Modelo económico}

La experiencia más larga de aplicación de blockchain es sin duda como respaldo de monedas como el bitcoin. Sin embargo, la gestión completa de la gobernanza en un sistema económico y su aplicación en actividades monetarias como sociales queda pendiente de establecer las bases de equivalencia para pagos, compras, acciones, aportaciones, comisiones entre todo tipo de operaciones -incluso a escala micro- guardando la contabilidad, ofreciendo confiabilidad y seguridad. La idea es central cuando se trata de lograr una economía circular, más humana y social, de mejorar la creación de comunidad y de dar valor a los intercambios.

Los costes operativos se reducen en paralelo al desarrollo de nuevos modelos de negocio y nuevos productos ligados a blockchain. Sería necesario el desarrollo de un mínimo de 'masa crítica' para poder desplegar las oportunidades que presenta el "efecto red" (Anderson, 2007): el efecto que se registra cuando el consumo de una persona tiene una influencia directa en la utilidad de otra u otras. A través de la interconectividad en red se produce el efecto de la conocida como 'larga cola' (Anderson, 2007) que permite la atención a un mercado 
de necesidades muy diversas, a través del coste marginal cero (Rifkin, 2014). El nuevo escenario sería una economía horizontal de la abundancia, gracias al coste marginal cero y a la participación de los consumidores en el ciclo productivo, a través del rol que la economía digital le asigna como prosumidor. La desintermediación incluirá nuevos actores en los ciclos productivos, la descentralización facilitará el acceso y la participación, y la confianza dará respaldo social al modelo económico.

Como valor añadido habría que mencionar un nuevo modelo de participación ya que, al desplegar el código de blockchain para que quede visible para los participantes, todo el proceso empresarial y su coherencia con las políticas y la regulación dependerán de la comunidad que lo respalde. En cualquier caso, una moneda local y un círculo económico basado en el valor de uso por encima del valor de compra favorece la economía local. Blockchain permitirá hacer el seguimiento a la producción local y a sus actividades, que se pueden valorar dentro de la economía circular.

\subsection{Economía circular y colaborativa}

La economía circular se apoyaría en una moneda local, que haría operativo el intercambio de valor entre la misma y los bancos de tiempo, cerrando las fugas del sistema, reforzando la eficiencia del modelo y generando valor al interior de la comunidad. Una cualidad de gran interés que aporta blockchain es la trazabilidad de los productos que puede contribuir tanto a la generación de un círculo económico como a la construcción de una marca local, con garantía de calidad de origen al permitir rastrear la procedencia de los productos ya que consumidores, intermediarios y administración podrán consultarla en cada momento. Este seguimiento se puede realizar no sólo para productos físicos sino también inmateriales como el valor, la reputación, o la información. En este sentido, habría que destacar iniciativas como chronobank.io / Chrono.Tech (Chronobank, 2020) que permite los intercambios de diferentes características y valor.

A la hora de -rentabilizar los recursos naturales, los culturales y las oportunidades que ofrecen para la economía y el emprendimiento social, blockchain será eficaz para gestionar la información, llevar la contabilidad y las equivalencias sobre las transacciones que se realicen, llevando la contabilidad de los intercambios y sus equivalencias, la monetización de creaciones artísticas y culturales, pero también la garantía de los derechos de autor al servir de registro de la autoría de creaciones, de forma inmodificable y verificable.

Para esta gestión, habría que diseñar un marco de coordinación público-privado desde un enfoque de la gobernanza de las políticas de empleo, la economía social y la innovación. La conjunción e implicación de los agentes sociales, económicos y políticos en el territorio necesita un modelo de intercambios para rentabilizar recursos y generar nuevos empleos. De cara al equilibrio en las tensiones existentes entre los diferentes actores y de dar respuesta a las necesidades de capital humano de las empresas, las acciones del proyecto se encaminan a lograr mayor conectividad y la experiencia del proyecto en blockchain puede buscar un equilibrio en el sistema.

\subsection{Empleos de la economía digital y local}

Los cambios económicos tanto en el mundo digital como en lo local han conducido a servicios y trabajos que se pueden desempeñar tanto en la proximidad como en el mundo digital precisan de nuevos modelos de pago, fiscalidad y reconocimiento ya que a menudo se desempeñan en pequeñas fracciones de tiempo, sin que haya sistemas de pago a escala micro que puedan recompensar la prestación de pequeños servicios, incluso en diferentes sectores de actividad, o el pago en moneda local.

Blockchain canaliza la economía colaborativa sin necesidad de recurrir a plataformas del tipo BlaBlaCar o AirBnB. Así entre particulares, se podrá alquilar o intercambiar servicios aplicando un sistema de micropagos que se gestionaría a través de blockchain aplicable también a automóviles o bicicletas gracias a dispositivos con GPS ligados a estos bienes de forma que queda constancia de la propiedad y de los desplazamientos (se convierte así también en un seguro antirrobo). Las transferencias económicas se pueden realizar en cualquier momento, en un sistema equivalente al de pago por móvil que sirve para el envío de remesas sin coste, sin esperas para la confirmación ni cantidades mínimas de transferencia.

La gestión económica a través de blockchain se aplicaría también con diferentes objetivos relacionados con la transición hacia la economía digital.

1. De una parte, la necesidad de modelos más ágiles y flexibles de pago acordes con los microservicios que tienen enormes dificultades para su contabilidad, monetarización y fiscalidad.

2. De otra, de cara a aflorar la economía sumergida producida por un modelo ineficiente de fiscalidad y cotizaciones dentro de la categoría de autónomos. La exigencia del alta de autónomos y de sus cotizaciones resulta disuasoria para quienes sólo obtienen pagos de muy poca cuantía.

3. Para el análisis de los sistemas de subsidios y su eficacia, ya que a través de blockchain se puede controlar si se registra duplicidad en las subvenciones o en los subsidios, así como la justificación y rendición de cuentas por parte de las personas beneficiarias.

Se puede lograr una mayor transparencia económica o una fiscalidad más completa y justa con sistemas que contemplen microunidades de medida para el pago, para trabajos y servicios, gracias a la garantía del exhaustivo sistema de contabilidad que puede gestionar blockchain. El análisis de las interacciones conduciría a establecer una equivalencia entre trabajos, tiempos, remuneración 
y acciones sociales. La determinación del valor de las mismas impulsaría las dinámicas de las transacciones, que resultarían más consolidadas. Fairfield (2014) defiende el papel de esta tecnología por sus contratos inteligentes y el papel que puede jugar para corregir los contratos abusivos.

Se integra en el círculo de la economía no sólo el valor de las contraprestaciones de ese régimen, sino también el de las acciones sociales que podrían equipararse al trabajo remunerado consideradas como capital social convertible en ayudas o beneficios para las personas y las asociaciones.

\subsection{Cláusulas sociales y reserva de mercado}

El modelo de contrataciones públicas ha cambiado de la búsqueda del bajo precio a una política más social, con la introducción de cláusulas sociales para promover el empleo en colectivos en riesgo de exclusión social. Se convierte así en una oportunidad específica la creación de empresas de inserción, cuya finalidad es la de la integración y formación de personas en situación de exclusión social, porque en muchos casos serán las beneficiarias de la contratación pública responsable. Se trata de hacer productivas actividades tradicionales aplicando la innovación y la capacitación de las personas más vulnerables ante el desempleo (mujeres con cargas familiares, personas desempleadas de larga duración, jóvenes, personas en riesgo de sinhogarismo, etc.).

En este sentido, hay que recordar que la Unión Europea (UE) centra sus políticas en las personas como la mejor fuente de la innovación, en un sistema en el que instituciones y ciudadanía pueden contribuir a la calidad de vida en las ciudades con la creación de laboratorios, impulsados por las TIC y las interacciones. La innovación social es un pilar del octavo programa marco de investigación e innovación de la UE, Horizonte 2020 (CE, 2020), que busca así dar respuestas a la escasez de recursos, a la salud, al cambio climático o al envejecimiento activo. Para ello, se ha marcado como objetivo que los países que la integran tengan que destinar el $3 \%$ de su PIB al gasto en investigación y desarrollo (I+D). En su estrategia Europa 2020, la UE ha identificado áreas de interés como son el empleo, la investigación, el desarrollo y la innovación $(\mathrm{I}+\mathrm{D}+\mathrm{i})$, el cambio climático, la educación y la exclusión social. Todas ellas coherentes con el planteamiento de las iniciativas de este proyecto.

La Estrategia Europea 2020 apuesta por la contratación con cláusulas sociales de cara a impulsar el crecimiento económico sostenible e integrador con un uso más eficiente de los fondos públicos y la participación de PYMES, las empresas de inserción social o las de la economía social en general. Estas políticas producen empleo y reducen los costes de los subsidios, eliminan subvenciones, dinamizan la economía local, y fomentan la responsabilidad social de las empresas y de los proyectos de economía social. Es un instrumento para generar políticas activas de empleo al considerar la relación calidad-precio de una forma más completa ya que las ofertas con muy bajo precio suelen generar después costes diferidos para el sector público porque en ellas se suelen registrar incumplimientos en materia medioambiental o social. Así se enfatiza el proceso de creación de valor público con un rediseño del ciclo de estas políticas, dentro de un modelo participativo en la definición del problema, de los objetivos, de la elección de las acciones y servicios, así como de su evaluación, a través del fomento de una ciudadanía activa.

El diseño blockchain que se propone aquí permitiría la aplicación directa de las cláusulas sociales en la contratación pública, al unificar criterios económicos y de valor aplicables de forma directa, como parte del consenso del blockchain. Sería un campo experimental para establecer criterios e indicadores para el cálculo de la reserva de mercado de la contratación pública, prevista en un 7\% del montante total de cada licitación, pero que está pendiente de desarrollo legislativo en España. La dificultad para establecer un cálculo para esta reserva se encuentra en el cambio del criterio de la 'oferta más favorable' a la de 'mayor interés público', que puede incluir aspectos que no sean cuantitativos. La práctica a través de blockchain puede incluir el estudio previo de las cláusulas y su cumplimiento, la obligación de cláusulas sociales en la subcontratación, la reserva para centros especiales de empleo o empresas de inserción, la revisión de la información sobre el cumplimiento de las condiciones como exclusión social o grado de discapacidad, todo ello como un consenso de creación del blockchain.

\section{Ciudadanía, servicios ciudadanos y Tercer sector}

Blockchain es un sistema de gran solidez y flexibilidad para la atención de los servicios ciudadanos ya que los participantes en las transacciones comparten y tienen acceso a los mismos registros no será necesaria la intermediación para acreditar los procedimientos relacionados con licencias, matrículas, o propiedad. Incluso se estima que la ciudadanía pueda prescindir de servicios legales, registros y notarías (Jiménez, 2018), así como de desplazamientos. Además, la tecnología reduciría la discrecionalidad administrativa en la gestión, así como la de los propios funcionarios ya que el procedimiento es auditable. Los datos y la información almacenados en la cadena de bloques son transparentes en el sistema, ya que quedan abiertos de forma permanente para verificación y supervisión de todos los participantes, pero también de forma inalterable por la estructura distribuida de la configuración de almacenamiento de los datos.

\subsection{Empoderamiento ciudadano, gestión de la identidad $y$ de accesos}

Blockchain permite que la gestión de la identidad y los datos de los ciudadanos sea personal, es decir, que no dependa de terceros ya que el modelo tecnológico contribuye a la construcción de una identidad digital propia e inalterable, un identificador que no podrá ser apropiado ni por particulares ni por instituciones. Así el control de la información personal dependerá de uno mismo ya 
que posibilita que cada persona sea la única propietaria de sus propios datos. Guy Zyskind et al. (2015) describen una base de datos personal descentralizada que garantiza a los usuarios la propiedad y el control de sus datos, sin necesidad de confianza en un tercero. Junto con el empoderamiento personal que todo ello supone, sería también una fuente de riqueza al participar en los beneficios que hasta ahora se apropian las corporaciones.

Además, el sistema evita las falsificaciones, fraudes y usurpación de la personalidad que se pueden registrar tanto a través de los documentos de identidad como de su manipulación física o virtual. Los documentos oficiales identitarios se consideran como un token o representación física de la información contenida en el ledger que en blockchain quedan vinculados sin necesidad de realizar diferentes trámites ante la administración.

Para facilitar la integración de colectivos que sufren la exclusión social supone la posibilidad de que un migrante que haya perdido sus documentos en su tránsito recupere su identidad. De la misma forma, podrían conseguirlo las personas sin hogar que sufren de la exclusión en el acceso a servicios públicos por falta de una dirección física o de registro en el censo. El cruce de información que realiza blockchain en diferentes bases de datos permite validar la identidad cuando faltan las clásicas pruebas de papel.

\subsection{Tercer sector}

Blockchain cuenta con menos experiencias dentro del Tercer sector, a pesar de sus claras ventajas y de los beneficios que aportaría a este tipo de organizaciones, debido a la dificultad de financiación y de desarrollo de esta tecnología. Dentro de la organización interna de asociaciones del Tercer Sector las ventajas y oportunidades son similares a las descritas para la administración en cuanto a su gobernanza: la gestión de la democracia interna, la votación de los socios, la gestión de los representantes que tiene un escenario de máxima transparencia y garantía.

Las donaciones y cuotas, las subvenciones que reciben estas organizaciones mejorarían de forma significativa ya que el sistema se adapta a los micropagos desde móvil aplicable a las campañas de sensibilización como sistema recaudatorio. Aplicado a campañas de crowdfunding facilitaría la devolución de los fondos si no se cumple el objetivo de la campaña. En el caso de que sí se cumpla, facilitaría el seguimiento del destino de los fondos por parte de los donantes de la misma forma que otros tipos de donaciones, por ejemplo, para campañas de recogida de alimentos o de gestión de grandes catástrofes. La información que aporta el sistema, realimentada en el círculo de gestión de la ONG, mejoraría de forma significativa ya que permite investigar, a través de la trazabilidad, los desvíos y fallos en la gestión respecto a los objetivos inicialmente planteados o bien la mejora del análisis de las necesidades. Se puede orientar a las alianzas y aportaciones de empresas dentro de sus programas de Responsabilidad Social Corporativa, por su garantía, pero también para el análisis de experiencias y buenas prácticas que se conviertan en escalables o bien en adaptables a otras áreas empresariales.

\section{Riesgos y efectos no contemplados}

Blockchain aparece con la promesa de una respuesta para los retos más importantes que hoy se plantean en la sociedad digital, la transparencia, la privacidad, la seguridad y el control, pero tiene todavía desafíos por resolver tanto en su desarrollo tecnológico y en su regulación. Blockchain ha irrumpido en la cultura digital mostrando ventajas objetivas, que quedan pendientes de su aplicación práctica, que despierta también desconfianza en muchos sectores porque al igual que ha ocurrido con otras transformaciones producidas por la digitalización, esta tecnología supone un cambio de mentalidad y de habilidades. En este sentido hay que añadir la necesidad de formación especializada para generalizar los sistemas de blockchain, y el fomento de una mayor cultura de la seguridad de los usuarios.

Para el análisis de la solidez y eficacia de blockchain, se ha desarrollado Blockbench (Wang, 2017), un sistema de comparación entre diferentes plataformas, en especial de las opciones de diseños, que funciona a través de las API y mide en términos generales y de componentes en términos de rendimiento, latencia, escalabilidad y tolerancia a las fallas. Dinh et al. (2017) han testado tres importantes blockchain para asegurar que estos sistemas aún están lejos de desplazar a los actuales en el tratamiento tradicional de datos. Por otra parte, $\mathrm{Xu}$ et al. (2017) proponen una clasificación y comparación de sistemas de configuración de blockchain en términos de calidad, pero también sobre el impacto de las principales decisiones sobre el diseño.

En el campo de la regulación legal y jurídica todavía hay riesgos de calado que están pendientes de una solución, como son: el marco legal sobre la naturaleza jurídica de las aplicaciones blockchain, la falta de reconocimiento del sistema como fuente de veracidad inmutable, la validez jurídica de la documentación almacenada, $\mathrm{y}$ de los instrumentos financieros emitidos por blockchain, el marco legal para los contratos inteligentes, así como la regulación sobre su uso como un registro de Internet de las Cosas (Sebastián, 2016). En su vertiente tecnológica, hay numerosos servicios de garantía con base en un blockchain como proof of existence (Proof of existence, 2000) que se convierte en la 'prueba de la existencia' de un documento o un contrato en un momento determinado del tiempo: un hash generado para el documento en cuestión, produce una transacción para ese documento que permite almacenar su código en blockchain, en el que consta fecha y hora, y es una prueba segura, pública y verificable de existencia, con un coste bajísimo. Dolader et al. (2017, pp. 39-40) mencionan también la seguridad que aporta la propia estructura de blockchain para recuperar el estado de la base de datos en cualquier punto del tiempo, mencionando como ejemplo la implementación de big data lakes que realiza la empresa Guardtime con su tecnología KSI basada en blockchain (GuardTime: Hadoop Big Data Lakes, 2017).

La imposibilidad técnica de eliminar o falsear cualquier registro en el histórico de las transacciones ejecutadas en un blockchain puede plantear también riesgos. Por ejemplo, el conocido como "derecho al olvido", 
reconocido en la legislación europea de protección de datos personales, que supone la eliminación - a petición de la persona afectada- de la información de cualquier ciudadano almacenada en cualquier tipo de formato, es técnicamente imposible de cumplir en un blockchain. Sebastián (2016) apunta la solución de sustituir el derecho a la "eliminación" por el derecho a la "imposibilidad de uso" de información personal por parte de terceros, a través de una solución encriptada de carácter automático. El riesgo no está solo en las imposibilidades técnicas derivadas de la naturaleza distribuida y criptográfica de blockchain, sino que, como todo sistema de software, es inevitable que puedan producirse errores en el diseño, la construcción o la configuración sobre el que se basa el blockchain.

Por lo tanto, para que blockchain consolide su capacidad disruptiva, la regulación del sistema será un elemento clave. El desafío va camino de resolverse, pero no la dificultad de impulsar una transformación de las administraciones e instituciones para aceptar que el sistema de tecnologías informáticas basado en blockchain puede estructurar la gestión pública y la compatibilidad en las interacciones con industrias y empresas, las operaciones de seguimiento y en general todos los contratos de garantía. De hecho, Tapscott (2016) menciona los obstáculos que se plantean ante la irrupción de blockchain, entre los que destaca la inmadurez de la sociedad así como de la propia tecnología, los sistemas de poder que tratarán de bloquear o controlar iniciativas de blockchain, la previsible pérdida de puestos de trabajo, la reducción progresiva de incentivos para la colaboración colectiva $y$, especialmente, en cuestiones de gobernanza sobre las que plantea cuestiones como la responsabilidad de un sistema o compañía descentralizada, el pago de impues- tos, el papel de los gobiernos o la posible existencia de naciones digitales.

En términos de coste, blockchain presenta la dificultad inicial de su implantación porque, aunque puede ofrecer un gran ahorro a las organizaciones, su implantación en términos económicos supone desembolsos muy elevados para la adopción de la tecnología, pero también por los cambios estructurales del paso de sistemas centralizados a descentralizados. Todavía más significativo puede llegar a ser el consumo energético necesario para mantener el sistema activo y los cálculos de su algoritmo. Por ejemplo, O’Dwyer y Malone (2014) estimaron que, en ese año, bitcoin consumía la misma cantidad de energía que toda Irlanda.

Al margen de las dificultades técnicas, operativas y regulatorias, lo cierto es que la aplicación de blockchain exige importantes cambios en los modelos de gestión, el liderazgo público y privado, e incluso plantea la necesidad de una nueva cultura ciudadana y democrática. En el campo económico producirá transformaciones importantes en la manera de hacer negocios, compartir información, y generar riqueza ya que los beneficios que obtienen los inversores, consumidores, empresarios y ciudadanos que tendrán la libertad de elegir, y también el control sobre sus datos. Todo ello entra en conflicto directo con el funcionamiento de las instituciones, gobiernos y el Estado, así como con los reglamentos, procesos políticos y costumbres por lo que puede representar un freno para la normalización.

En este momento, la importancia de las transformaciones digitales y tecnológicas se encuentra en la experimentación para conocer su capacidad transformadora y el verdadero impacto que produce en los aspectos sociales, políticos y económicos, así como, especialmente, en la vida de las personas.

\section{Referencias}

Alonso, A. y Lafuente, A. (2011). Gobernanza electrónica y procomún digital. Nomads. Mediterranean Perspectives, 2. Recuperado de: http://www.theoria.eu/nomads/02/alonso_lafuente_es.pdf

Anderson, C. (2007). La economía long tail. Barcelona: Urano.

Arteaga Moralejo, J. (2018). Blockchain en procesos de participación ciudadana: innovando desde la práctica en el Ayuntamiento de Alcobendas. En Güemes, C., Resina, J. y Cruz-Rubio, C. (Eds.), Participación ciudadana: Experiencias inspiradoras en España. Madrid: Centro de Estudios Políticos y Constitucionales.

Brandom, R. (2019, enero 9). Why the Ethereum Classic hack is a bad omen for the blockchain. The Verge, 2019. Recuperado de: https://www.theverge.com/2019/1/9/18174407/ethereum-classic-hack-51-percent-attack-double-spend-crypto

Calderón, C. y Lorenzo, E. (2010). Open Government: Gobierno Abierto. Jaén; Algón Editores.

CEOE (Confederación Española de Organizaciones Empresariales) (2014). Prioridades y propuestas empresariales. VIII Legislatura europea, 2014-2019. Recuperado de: https://contenidos.ceoe.es/resources/image/prioridades_propuestas_ empresariales_elecciones_pe_2014.pdf

ChronoBank / Chrono.Tech (2020). https://chrono.tech/

Comisión Europea (2014). HORIZON 2020 en breve El Programa Marco de Investigación e Innovación de la Unión Europea. Luxemburgo: Oficina de Publicaciones de la Unión Europea.

De Filippi, P. (2017). What Blockchain Means for the Sharing Economy. Harvard Business Review. Recuperado de: https://hbr. org/2017/03/what-blockchain-means-for-the-sharing-economy

Dinh, T. T. A., Wang, J., Chen, G., Liu, R., Ooi, B.C. y Tan, K.-L. (2017). BlockBench: A Framework for Analyzing Private Blockchains. SIGMOD'17. Recuperado de: https://arxiv.org/abs/1703.04057

Dolader Retamal, C., Bel Roig, J. y Muñoz Tapia, J. L. (2017). La blockchain: fundamentos, aplicaciones y relación con otras tecnologías disruptivas. Economía industrial, 405, 33-40. Recuperado de: http://www.mincotur.gob.es/Publicaciones/ Publicacionesperiodicas/EconomiaIndustrial/RevistaEconomiaIndustrial/405/DOLADER,\%20BEL\%20Y\%20 MU\%C3\%91OZ.pdf

EUBlockchain Observatory and Forum (2018). Blockchain for Government and Public Services. Recuperado de: https://www. eublockchainforum.eu/sites/default/files/reports/eu_observatory_blockchain_in_government_services_v1_2018-12-07.pdf 
Fairfield, J. (2014). Smart Contracts, Bitcoin Bots and Consumer Protection. Washington \& Lee Law Review Online, 71(2), $35-50$. Recuperado de: http://scholarlycommons.law.wlu.edu/wlulr-online/vol71/iss2/3

Garcés Noblecía, R. (2011). Formación Ciudadana en una República Electrónica. Documento del Cuarto Foro Internacional de Derechos Humanos y Tecnologías de la Información y la Comunicación. https://repositoriodigital.ipn.mx/ handle/123456789/3910

GitHub (2020). Built for developers. https://github.com/

Ibáñez Jiménez, J. W. (2018). Blockchain: primeras cuestiones en el ordenamiento español. Madrid: Dykinson.

IrisGuard (2020). Why IrisGuard? https://www.irisguard.com/

Jun, M. (2018). Blockchain government - a next form of infrastructure for the twenty-first century. Journal of Open Innovation: Technology, Market, and Complexity, 4. http://dx.doi.org/10.1186/s40852-018-0086-3

O’Dwyer, K. J. y Malone, D. (2014). Bitcoin Mining and its Energy Footprint ISSC 2014 / CIICT 2014, Limerick, June $26-27$. Recuperado de: http://karlodwyer.com/publications/pdf/bitcoin_KJOD_2014.pdf

Preukschat, A. (2017). Los fundamentos de la tecnología blockchain. En A. Preukschat, (Coord.), Blockchain: la revolución industrial de internet. Barcelona: Gestión 2000.

Proof of existence (2020) Certify documents https://www.proofofexistence.com/

Raymond, E. S. (2001). Cómo convertirse en hacker. Recuperado de: http://biblioweb.sindominio.net/telematica/hacker-como. html

Rifkin, J. (2014). La sociedad de coste marginal cero. Barcelona: Paidós.

Sebastián Cermeño, J. (2016). Blockchain in financial services: Regulatory landscape and future challenges for its commercial application. BBVA Research, Working Paper 16/20. Recuperado de: https://www.bbvaresearch.com/wp-content/ uploads/2016/12/WP_16-20.pdf

Stallman, R. M. (2004). Software libre para una sociedad libre. Madrid: GNU Press.

Tapscott, D. y Tapscott, A. (2016). La revolución Blockchain. Barcelona: Deusto.

The GovLab (2017). About CrowdLaw: Online Public Participation in Lawmaking. Recuperado de: https://crowd.law/crowdlawaf1a9e1c9455

Voelker, A. (2012). GitLaw: GitHub for Laws and Legal Documents - a Tourniquet for American Liberty. Recuperado de: https:// abevoelker.com/gitlaw-github-for-laws-and-legal-documents-a-tourniquet-for-american-liberty/

Wang, J. (2017). Blockbench. A Framework for Analyzing Private Blockchains. Social Science Research Network, 2580664 Recuperado de: https://www.comp.nus.edu.sg/ dbsystem/blockbench/index.html

Wright, A. y De Filippi, P. (2015). Decentralized blockchain technology and the rise of Lex Cryptographia. http://dx.doi. org/10.2139/ssrn.2580664

Xu, X., Weber, I., Staples, M., Zhu, L., Bosch, J., Bass, L., Pautasso, C. y Rimba, P. (2017). A taxonomy of Blockchain-based systems for architecture design. ICSA'17: IEEE International Conference on Software Architecture. Gothenburg, Sweden, April 2017. http://dx.doi.org/10.1109/ICSA.2017.33

Zyskind, G., Nathan, O. y Petland, A. 'Sandy' (2015). Decentralizing Privacy: Using Blockchain to Protect Personal Data. IEEE Security and Privacy Workshops (SPW), 2015. San Jose, CA, USA, 180-184. http://dx.doi.org/10.1109/SPW.2015.27 